\title{
Antitumor Effect of Vaccinia Virus Double Recombinant Strains Expressing Genes of Cytokine GM-CSF and Oncotoxic Peptide Lactaptin
}

\author{
G.V. Kochneva, O.A. Koval, E.V. Kuligina, A.V. Tkacheva \\ and V.A. Richter
}

\begin{abstract}
In this study, the double recombinant vaccinia viruses were generated those express exogenous proteins: human granulocyte-macrophage colony-stimulating factor (GM-CSF) and the antitumor protein lactaptin in secreted and nonsecreted forms. We observed that recombinant VV-GMCSF-Lact with nonsecreted lactaptin exerted stronger cytotoxic activity than others in MDA-MB-231, BT-549 and BT-20 breast cancer cells with calculated $\mathrm{CD}_{50}$ of 0.005; 0.004 и 0.00083 PFU/cell correspondently. Strain VV-GMCSF-Lact also exhibited highest lytic activity in lung cancer cells H1299 and epidermoid carcinoma cells A-431. Normal MCF10A cells and diploid embryonic lung human cells LECH-240 were resistant to all recombinant vaccinia viruses. Strain VV-GMCSF-Lact showed the highest index of tumor selectivity in pairs normal/cancer cells: MCF10A/MDA-MB-231 (>2000) and LECH-240/H1299 (190). By flow cytometry, we demonstrated that all recombinants induced apoptosis in treated cancer cells but the rate of annexin $\mathrm{V}$-positive cells was higher after treatment with VV-GMCSF-Lact than others. Thus nonsecreted lactaptin expression increased the toxicity of recombinant virus to cancer cells in the best way. It is likely that lactaptin expression inside the treated cells (without secretion outside)
\end{abstract}

G.V. Kochneva ( ()

State Research Center of Virology and Biotechnology "Vector", Novosibirsk, Russia e-mail: kochneva@vector.nsc.ru

O.A. Koval · E.V. Kuligina · A.V. Tkacheva · V.A. Richter

Institute of Chemical Biology and Fundamental Medicine SB RAS, Novosibirsk, Russia

e-mail: o.koval@niboch.nsc.ru

E.V. Kuligina

e-mail: kuligina@niboch.nsc.ru

A.V. Tkacheva

e-mail: tkacheva_av@mail.ru

V.A. Richter

e-mail: richter@niboch.nsc.ru

(C) The Author(s) 2018

K.V. Anisimov et al. (eds.), Proceedings of the Scientific-Practical Conference

"Research and Development - 2016”, https://doi.org/10.1007/978-3-319-62870-7_61 
intensifies apoptosis and as a consequence promotes the progression of apoptotic cells to secondary necrotic cells. These results demonstrate that recombinant VV-GMCSF-Lact has good oncolytic potential and stimulate further investigation of its anticancer activity in human tumor models in vivo and to use it in the development of anticancer therapeutic agents.

Keywords Recombinant vaccinia virus - Breast cancer cells $\cdot$ Apoptosis Lactaptin $\cdot$ GM-CSF $\cdot$ SCID mice $\cdot$ Tumor growth inhibition

Vaccinia virus (VACV) possesses many unique properties that place this virus at a leading position in molecular biology and genetic engineering. The ability to kill cancer cells is one of the fundamental biological properties of VACV, and was first reported by Levaditi C. and Nicolau S. in 1923 in the Annals of the Pasteur institute [16, p. 20]. Subsequent studies confirmed the oncolytic activity of VACV [14, p. 360]. However, over the next few decades' researchers tried to avoid complications caused by introducing the infectious virus into human organism. Progress in genetic engineering allowed changing the biological properties of VACV in a wide range, and this led to a surge of interest to oncolytic abilities of genetically modified VACV. Detailed analysis of whole bulk of the studies with genetically modified oncolytic VACV, some of which are under clinical trials, recently was published in several comprehensive reviews $[4$, p. $210 ; 5$, p. $7 ; 1$, p. 191].

The rational construction of a therapeutic VACV could be done using a virulent attenuated VACV strain with deletions of $t k$ and $v g f$ genes that would selectively target tumor cells without decreasing its oncolytic capacity [11, p. 2]. Two transgenes could be simultaneously inserted into the VACV genome to enhance the therapeutic efficacy of recombinant VACV-the gm-csf gene and the gene of cytotoxic protein lactaptin.

Lactaptin is a fragment of human milk kappa-casein (residues 57-134) that induces the death of cultured cancer cells. A recombinant analog of lactaptin, RL2, containing the complete amino acid sequence of lactaptin and corresponding to 23157 of human kappa-casein, effectively induces apoptotic death in various mouse and human tumor cells (including breast tumor cells and primary endometrial cells) and has no effect on the viability of non-malignant mesenchymal stem cells (MSCs) [12, p. $178 ; 8$, p. $2467 ; 2$, p. $79 ; 10$, p. 345]. RL2-induced apoptosis is accompanied by downregulation of BCL-2, activation of the executor caspase-3 and -7 and apoptotic fragmentation of DNA [9, p. 1]. The insertion of lactaptin sequence as a transgene into the deletion of $v g f$ gene could attenuate the virulence of recombinant VACV against non-transformed cells as well as enhance its cytotoxic activity against cancer cells.

Here, we exploited VACV L-IVP strain that was used for anti-smallpox vaccination in Russia up to 1980 [13, p. 1; 16, p. 1]. Thus, L-IVP has a good medical history in Russia, which could provide advantages in clinical trials of new 
L-IVP-based recombinant strains. We have previously demonstrated that genetically unmodified L-IVP possesses natural antitumor activity towards human and murine tumors [16, p. 1]. Recombinant VV-GMCSF-S1/3 in which the virus $t k$ gene is inactivated by insertion of the human gm-csf gene was engineered earlier [3, p. 9]. This VV-GMCSF-S1/3 strain was used as a recipient for insertion of additional RL2 transgene into the deleted $v g f$ gene region. We conducted a comparative study of both nonsecreted and secreted forms of lactaptin expressed as a part of a recombinant virus.

The objectives of this study were to generate new double recombinant VACV L-IVP strains expressing human GM-CSF and secreted or nonsecreted RL2 and to analyze its antitumor potential in vitro to choose the most promising construct for further research in vivo.

Recombinant VACVs were obtained via the transient dominant selection technique with the use of the puromycin resistance (Pat) gene as a selective marker [7, p. 4]. We constructed three recombinant strains containing a gm-csf gene insertion and different variants of lactaptin gene (RL2). Recombinant structures are shown in Fig. 1a. The VV-GMCSF-Lact strain encodes a nonsecreted form of RL2 that is produced only inside the infected cell [7, p. 4]. Strain VV-GMCSF-S(long)-Lact encodes chimeric RL2 protein with the signal peptide (MWLQSLLLLGTVACSIS) and the first 15 amino acids of GM-CSF (long signal sequence, S(long)) ligated to the $\mathrm{N}$ end. In the VV-GMCSF-S-Lact strain lactaptin presented as a chimera with a shorter leader fragment of GM-CSF, which does not contain the $15 \mathrm{~N}$-terminal amino acids of the protein (signal sequence, S). Strains VV-GMCSF-S(long)-Lact and VV-GMCSF-S-Lact encode secreted forms of RL2 protein due to the presence of the GM-CSF leader peptide. We used two variants of GM-CSF leader peptide in order to clarify the need for the presence of downstream GM-CSF protein sequence to improve the oncotoxic properties of RL2. By the same method the control recombinant VV-GMCSF-dGF containing the GM-CSF transgene in the $t k$ gene deletion and additional deletion of the $v g f$ gene was constructed (Fig. 1a). This control recombinant VV-GMCSF-dGF provided our study with the correct estimation of the double recombinants oncolytic activity enhancement.

The structure of recombinant viruses was confirmed by both PCR assays and DNA sequencing of the $t k$ and $v g f$ loci. Specific primer positions are depicted in Fig. 1a. We observed that all double recombinants and control recombinant VV-GMCSF-dGF produced a 1760 b.p. fragment in the $t k$ gene region that corresponded to the $g m$ - $c s f$ gene sequence whereas DNA of the parental VACV L-IVP strain produced a 414 b.p. fragment (Fig. 1b). Using primers flanking the VGF region we amplified fragments of 710; 836; 791 b.p. using DNA of the recombinants VV-GMCSF-Lact; VV-GMCSF-S(long)-Lact; VV-GMCSF-S-Lact and a fragment of 423 b.p. using DNA of the VV-GMCSF-dGF, corresponding to different variants of lactaptin gene insertion and $v g f$ gene deletion, respectively. A fragment of 584 b.p. was amplified from the DNA of the parental L-IVP strain using Up35 and Apa-L22 primers [7, p. 5]. 
(a)

\section{Construction and verification of oncolytic VACVs}

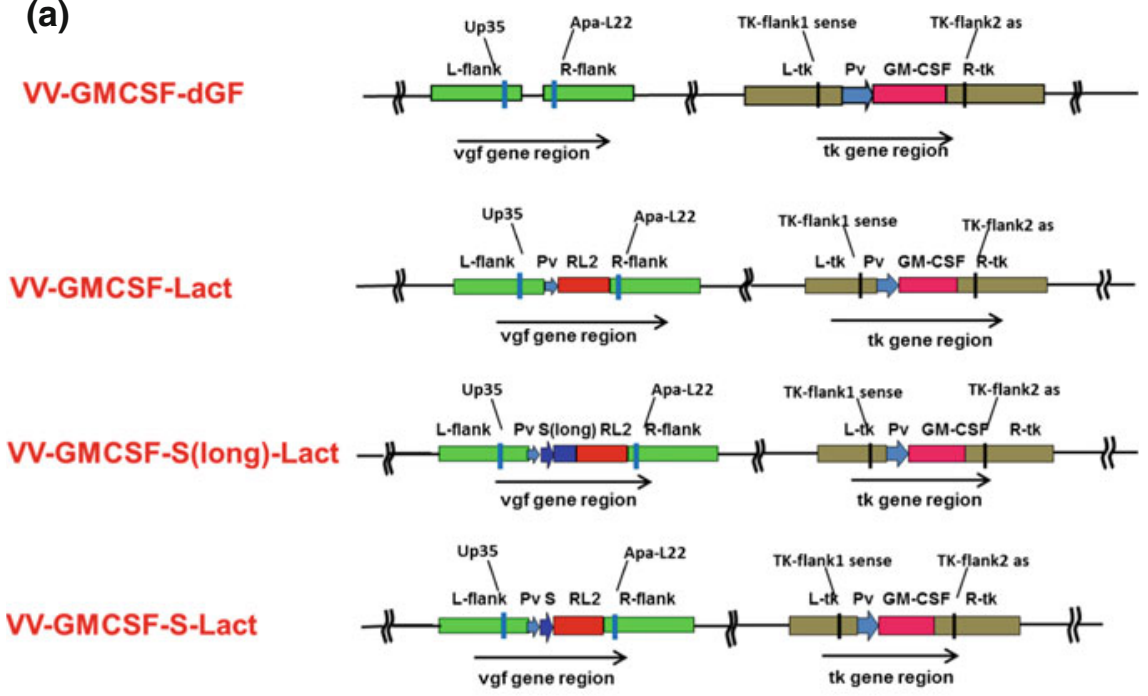

(b)

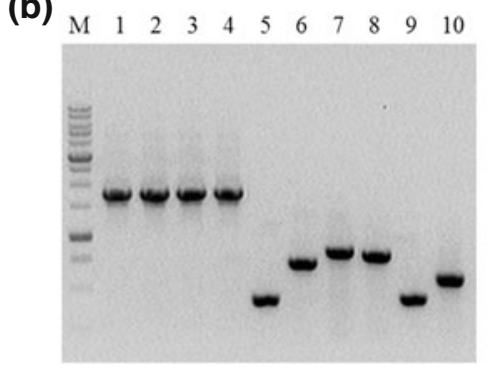

Fig. 1 Verification of recombinant VACVs structure. a schematic view of virus genomes with primer positions indicated. $P v$ VACV promoter; $S$ GM-CSF signal peptide sequence; $S$ (long) $\mathrm{S}+\mathrm{GM}-\mathrm{CSF}$ gene fragment (long signal); L-flank and $R$-flank sequences, flanking $v g f$ gene [6]; $L-t k$ and $R-t k$ sequences, flanking $t k$ gene [3]. b PCR identification of recombinant VACVs DNA with primers $T K$-flank1 sense and TK-flank 2 as (Lanes 1-5) and with primers Up35 x Apa-L22 (Lanes 6-10). Lanes 5 and 10-wild-type VACV (L-IVP); 1 and 6-VV-GMCSF-Lact; 2 and 7 VV-GMCSF-S(long)-Lact; 3 and 8-VV-GMCSF-S-Lact; 4 and 9-VV-GMCSF-dGF; 3 and 6 VV-GMCSF-Lact. $M$-DNA molecular weight marker

The cytotoxic activity of recombinant VACVs in vitro was analyzed using XTT assay as described previously [6, p. 737] and measured as the 50\% cytotoxic dose $\left(\mathrm{CD}_{50}\right)$, the virus concentration causing death of $50 \%$ of cells. Seven tumor cell lines of various origins were used to investigate the oncolytic activity of recombinant VACVs: breast cancer carcinomas BT-20, BT-549, MDA-MB-231, MCF-7, lung carcinoma A-549, non-small lung cell cancer H1299 and epidermoid carcinoma A-431. Summary results are presenting in Fig. 2. Breast carcinoma MCF-7 
cells were more resistant to recombinant viruses than the other breast cancer cells. MCF-7 cells are the only estrogen-dependent breast cancer cells, in contrast to MDA-MB-231, BT-549 and BT-20 and possibly, lack of estrogen in cell culture medium reduces the malignancy potential of these cells, and together with it the replicative virus activity. Since our recombinant VACVs contain the inactivated $t k$ and $v g f$ genes, they are sensitive to the presence of precursors in DNA synthesis and proliferation rate of infected cells. In conditions of estrogen deficiency, MCF-7 cells are inferior in these parameters estrogen-independent cultures and their lysis requires 5-50 times more virus.

We observed that recombinant VV-GMCSF-Lact exerted stronger cytotoxic activity than others in MDA-MB-231, BT-549 and BT-20 breast cancer cells with calculated $\mathrm{CD}_{50}$ of $0.005 ; 0.004$ и $0.00083 \mathrm{PFU} /$ cell correspondently. Strain VV-GMCSF-Lact also exhibited the highest lytic activity in lung cancer cells H1299 and epidermoid carcinoma cells A-431 (Fig. 2). Thus, nonsecreted lactaptin expression increased the toxicity of recombinant virus to cancer cells in the best way.

Tumor selectivity of recombinant VACVs was investigated using two noncancer cell lines: MCF 10A normal epithelial breast cells and LECH-240 diploid embryonic cells of human lung. As given in Fig. 2, the $\mathrm{CD}_{50}$ of recombinant strains, including the control recombinant VV-GMCSF-dGF, was significantly lower than the same dose for diploid and normal cells $(P<0.01)$. In the case of normal

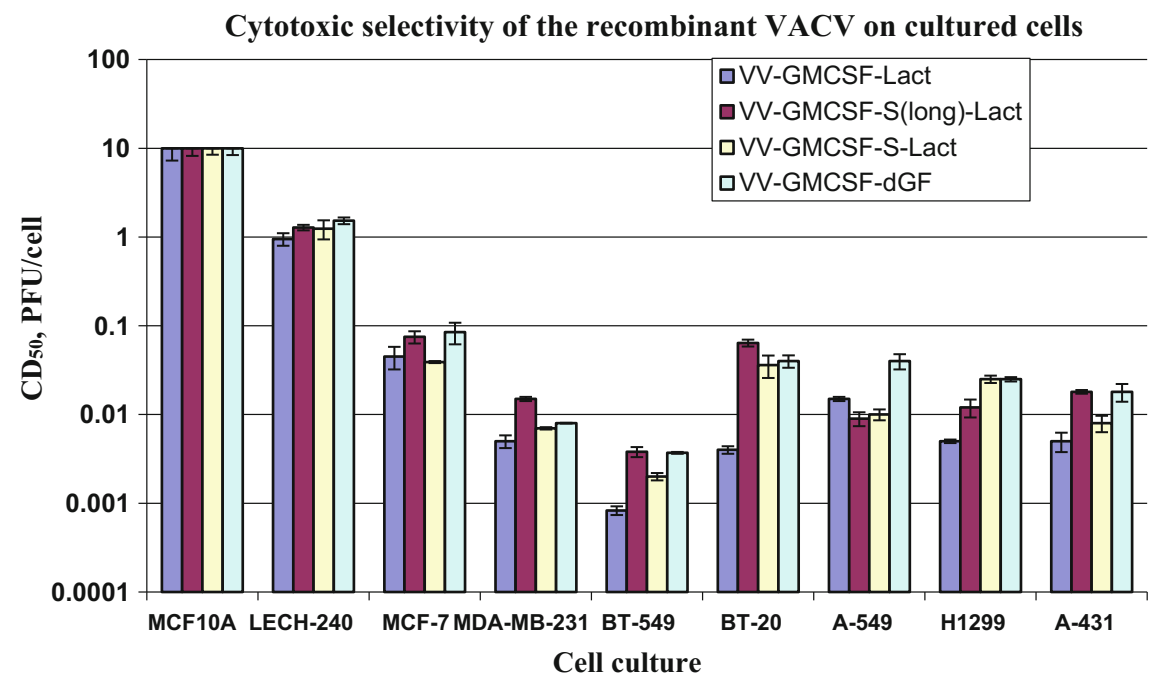

Fig. 2 Oncolytic and cytotoxic activities of recombinant VACV strains in vitro. Cells were grown in 96-well plates, infected with recombinant virus doses ranging from 0.001 to $10.0 \mathrm{PFU} / \mathrm{cell}$ (sequential tenfold dilutions) and incubated for $72 \mathrm{~h}$. The $50 \%$ cytotoxic dose $\left(\mathrm{CD}_{50}\right)$ was determined for each cell line by an XTT assay. Data are expressed as mean $\pm \mathrm{SE}$ 
Table 1 Comparison of cytotoxic activities of recombinant VACVs in two pairs of cells: normal breast epithelium MCF10A/breast carcinoma MDA-MB-231 and diploid embryonic lung LECH-240/lung cancer H1299

\begin{tabular}{l|l|l|l|l}
\hline \multirow{2}{*}{ Cell culture } & $\mathrm{CD}_{50}\left(\mathrm{PFU} /\right.$ cell $\left.^{\mathrm{a}}\right)$ & \multicolumn{4}{l}{} \\
\cline { 2 - 5 } & VV-GMCSF-Lact & VV-GMCSF-S-Lact & $\begin{array}{l}\text { VV-GMCSF- } \\
\text { S(long)-Lact }\end{array}$ & VV-GMCSF-dGF \\
\hline MCF10A & $>10$ & $>10$ & $>10$ & $>10$ \\
\hline MDA-MB-231 & 0.005 & 0.007 & 0.015 & 0.008 \\
\hline Selectivity index & $>2000$ & $>1400$ & $>650$ & $>1250$ \\
\hline LECH-240 & 0.95 & 1.24 & 1.28 & 1.53 \\
\hline H1299 & 0.005 & 0.012 & 0.025 & 0.025 \\
\hline Selectivity index & 190 & 103 & 51 & 61 \\
\hline
\end{tabular}

${ }^{a}$ The virus titer was determined by plaque assay and $\mathrm{CD}_{50}$ expressed as number of plaque forming units (PFU) per cell

${ }^{\mathrm{b}}$ Selectivity index was calculated for each virus as the ratio of $\mathrm{CD}_{50}$ values for normal and tumor cells

mammary epithelial MCF10A cells, we were unable to accurately determine the $50 \%$ cytotoxic dose for any of the virus strains as the maximum dose tested of 10 $\mathrm{PFU} /$ cell caused no toxic effect. The tumor selectivity index of recombinant viruses was calculated in pair MCF 10A/MDA-MB-231 or normal/cancer breast cells. The index value for all recombinant VACVs including control variant VV-GMCSF-dGF was more than 650 (Table 1) with the highest rate for VV-GMCSF-Lact strain (>2000).

Among all recombinant VACVs strain VV-GMCSF-Lact has a significantly higher cytotoxicity against H1299 lung cancer cells $(P<0.05)$. We calculated selectivity indexes paired cultures of lung embryo and human lung cancer cellsLECH-240/H1299 (Table 1). In this case, the value of selectivity indexes was lower than that of normal breast cells, specifically 190 in case of strain VV-GMCSF-Lact (Table 1). Apparently, this is due to the fact that embryonic cells have a relatively high proliferation activity, which, to some extent, makes them similar to cancer and increases the replicative activity of vaccinia virus recombinants. Recombinant VV-GMCSF-S(long)-Lact showed the lowest index of selectivity in relation to lung tumor cells (Table 1).

\section{Features of Apoptosis and Necrosis Induced by Recombinant VACVs}

The type of cancer cell death induced by newly constructed recombinant OVs coding the proteins with specific biological activity cannot be predicted precisely. We investigated apoptosis through the flow cytometry of plasma membrane phosphatidylserine exposure by using the BD Pharmigen Apoptosis Detection Kit (BD Biosciences). Staining with annexin V-FITC and propidium iodide (PI) allows separating apoptotic and necrotic cells. Phenotype annexin V+/PI- corresponds to 

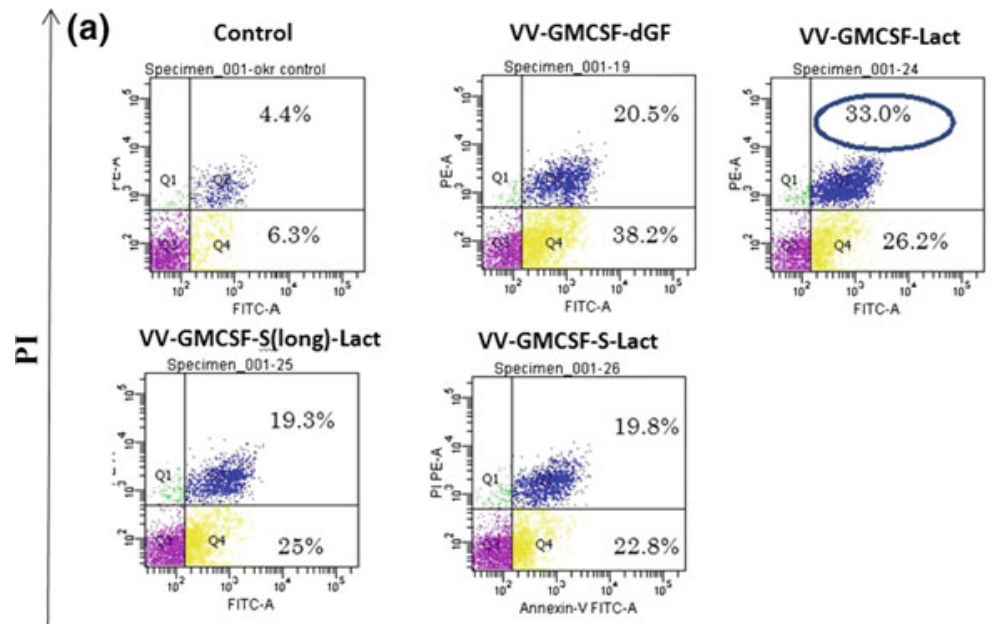

Annexin V-FITC

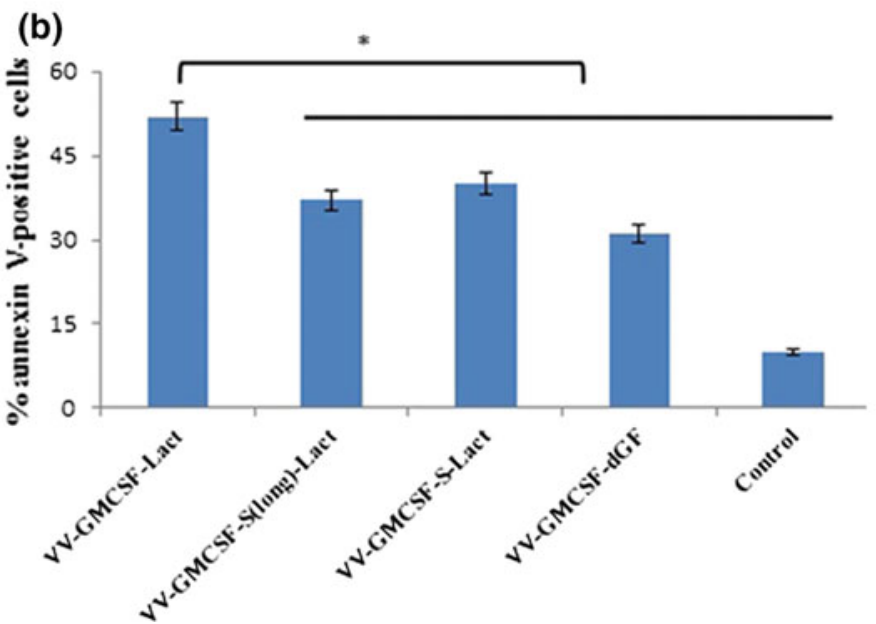

Fig. 3 Features of apoptosis of the MDA-MB-231 cells infected with recombinant VACVs. a MDA-MB-231 cells were treated with recombinant VACVs (1.5 PFU/cell) or with saline (control) for $48 \mathrm{~h}$ and then cells were harvested with trypsin and stained with annexin V-FITC and propidium iodide (PI) according to the manufacturer's protocol. Cell populations with the annexin $\mathrm{V}^{-} / \mathrm{PI}^{-}$phenotype $(\mathrm{Q} 3)$ were designated as living cells, annexin $\mathrm{V}^{+} / \mathrm{PI}^{-}(\mathrm{Q} 4)$ as apoptotic cells, and annexin $\mathrm{V}^{+} / \mathrm{PI}^{+}(\mathrm{Q} 2)$ as secondary necrotic cells. b Bar graph summarized the percentage of apoptotic cells from three independent experiments $(* p<0.05)$

cells in a state of apoptosis, annexin $\mathrm{V}-/ \mathrm{P}+$ - cells in a state of necrosis and annexin $\mathrm{V}+/ \mathrm{PI}+$ - cells in the state of late apoptosis or secondary necrosis [15, p. 180]. 
MDA-MB-231 cancer cells were treated with recombinant VACVs (1.5 $\mathrm{PFU} / \mathrm{cell}, 48 \mathrm{~h}$ ) and then were analyzed for apoptosis and necrosis by flow cytometry. We observed that the apoptosis rate of virus-treated cells dramatically increased compared with nontreated cells and that strain VV-GMCSF-Lact induced more extensive cell death than other recombinants analyzed (Fig. 3a). In VV-GMCSF-Lact treated cells, the population of secondary necrotic cells annexin $\mathrm{V}^{+} / \mathrm{PI}^{+}$was consistently higher $(33 \%)$ than that in VV-GMCSF-S(long)-Lact or VV-GMCSF-S-Lact treated cells (25 and $22.8 \%$ correspondently) whereas early apoptotic populations annexin $\mathrm{V}^{+} / \mathrm{PI}^{-}$differed slightly. Since apoptotic cells that have compromised plasma membrane integrity become subject to secondary necrosis (the phase that occurs after apoptosis in vitro), we analyzed the pooled annexin $\mathrm{V}^{+}$population which was larger in VV-GMCSF-Lact treated cells (Fig. 3b). It is likely that lactaptin expression inside the treated cells (without secretion outside) intensifies apoptosis and as a consequence promotes the progression of apoptotic cells to secondary necrotic cells. We found also that treatment of MDA-MB-231 cells with VV-GMCSF-Lact increased the size of the cell population with active caspase-3 and -7 in comparison with control VV-GMCSF-dGF [7, p. 9]. Thus, VACV-dependent expression of apoptosis-inducing proteins may promote the alteration of the route of death of infected cancer cells to apoptosis. This could be a helpful strategy to reinforce the oncolytic potential of recombinant VACVs.

\section{Conclusions}

We constructed three recombinant VACVs coding human GM-CSF and different forms of oncotoxic peptide lactaptin, secreted and nonsecreted. To estimate the contribution of lactaptin to the oncolytic activity of recombinant VACVs, we also constructed a recombinant coding GMCSF without the $v g f$ gene. Our results showed that normal MCF10A cells and diploid embryonic lung human cells LECH-240 were resistant to recombinant VACVs. Among the investigated cancer cell lines, breast cancer cell lines MDA-MB-231 and BT-549 were the most sensitive to recombinant viruses. The VV-GMCSF-Lact strain exhibited significantly higher lytic activity in the majority of tumor cells tested. It also showed the highest index of tumor selectivity in pairs normal/cancer cells: MCF10A/MDA-MB-231 (>2000) and LECH-240/H1299 (190). We observed that the apoptosis rate of VV-GMCSF-Lact treated cancer cells significantly increased compared with other recombinants analyzed. Taking into account our results, it seems promising to further investigate the oncolytic action of VV-GMCSF-Lact in human tumor models in vivo and to use it in the development of anticancer therapeutic agents.

Acknowledgements Research is carried out with the financial support of the state represented by the Ministry of Education and Science of the Russian Federation. Agreement no. 14.604.21.0057 27. Jun 2014. Unique project identifier: RFMEFI60414X0057. 


\section{References}

1. Chan, W.M., McFadden, G.: Oncolytic poxviruses. Annu. Rev. Virol. 1, 191-214 (2014)

2. Fomin, A.S., Koval, O.A., Semenov, D.V., Potapenko, M.O., Kuligina, E.V., Kit, I.I., Richter, V.A.: The analysis of biochemical markers of MCF-7 cells apoptosis induced by recombinant analog of lactaptin. Russ. J Bioorg. Chem. 38, 77-82 (2012)

3. Grazhdantseva, A.A., Sivolobova, G.F., Tkacheva, A.V., Gileva, I.P., Kuligina, E.V., Rikhter, V.A., Kochneva, G.V.: High-effective production of biologically active secreted human granulocyte macrophage colony-stimulating factor by recombinant vaccinia virus. Appl. Biochem. Microbiol. 52(7), 7-13 (2016)

4. Kim, M.: Replicating poxviruses for human cancer therapy. J. Microbiol. 53(4), 209-218 (2015)

5. Kochneva, G.V., Sivolobova, G.F., Yudina, K.V., Babkin, I.V., Chumakov, P.M., Netesov, S.V.: Oncolytic poxviruses. Mol. Genet. Microbiol. Virol. 27(1), 7-15 (2012)

6. Kochneva, G.V., Babkina, I.N., Lupan, T.A., Grazhdantseva, A.A., Iudin, P.V., Sivolobova, G.F., Shvalov, A.N., Popov, E.G., Babkin, I.V., Netesov, S.V., Chumakov, P.M.: Apoptin enhances the oncolytic activity of vaccinia virus in vitro. Mol. Biol. 47(5), 733-742 (2013)

7. Kochneva, G., Sivolobova, G., Tkacheva, A., Grazhdantseva, A., Troitskaya, O., Nushtaeva, A., Tkachenko, A., Kuligina, E., Richter, V., Koval, O.: Engineering of double recombinant vaccinia virus with enhanced oncolytic potential for solid tumor virotherapy. Oncotarget. (2016). doi:10. 18632/oncotarget.12367. https://www.ncbi.nlm.nih.gov/pubmed/27708236

8. Koval, O.A., Fomin, A.S., Kaledin, V.I., Semenov, D.V., Potapenko, M.O., Kuligina, E.V., Nikolin, V.P., Nikitenko, E.V., Richter, V.A.: A novel pro-apoptotic effector lactaptin inhibits tumor growth in mice models. Biochimie. 94, 2467-2474 (2012)

9. Koval, O.A., Tkachenko, A.V., Fomin, A.S., Semenov, D.V., Nushtaeva, A.A., Kuligina, E.V., Zavjalov, E.L., Richter, V.A.: Lactaptin induces p53-independent cell death associated with features of apoptosis and autophagy and delays growth of breast cancer cells in mouse xenografts. PLoS one 9, e93921 (2014)

10. Koval, O.A., Sakaeva, G.R., Fomin, A.S., Nushtaeva, A.A., Semenov, D.V., Kuligina, E.V., Gulyaeva, L.F., Gerasimov, A.V., Richter, V.A.: Sensitivity of endometrial cancer cells from primary human tumor samples to new potential anticancer peptide lactaptin. J. Cancer Res. Ther. 11, 345-351 (2015)

11. McCart, A., Bartlett, D., Moss, B.: Combined growth factor-deleted and thymidine kinase-deleted vaccinia virus vector. US Patent 7208313. (2007)

12. Semenov, D.V., Fomin, A.S., Kuligina, E.V., Koval, O.A., Matveeva, V.A., Babkina, I.N., Tikunova, N.V., Richter, V.A.: Recombinant analogs of a novel milk pro-apoptotic peptide, lactaptin, and their effect on cultured human cells. Protein J. 29, 174-180 (2010)

13. Shvalov, A.N., Sivolobova, G.F., Kuligina, E.V., Kochneva, G.V.: Complete genome sequence of vaccinia virus strain L-IVP. Genome Announc. 4, e00372-16 (2016)

14. Thorne, S.H., Hwang, T.H., Kirn, D.H.: Vaccinia virus and oncolytic virotherapy of Cancer. Curr. Opin. Mol. Ther. 7(4), 359-365 (2005)

15. Vermes, I., Haanen, C., Reutelingsperger, C.: Flow cytometry of apoptotic cell death. J Immunol. Methods 243, 167-190 (2000)

16. Zonov, E., Kochneva, G., Yunusova, A., Grazhdantseva, A., Richter, V., Ryabchikova, E.: Features of the antitumor effect of vaccinia virus Lister Strain. Viruses 8, 20 (2016) 
Open Access This chapter is licensed under the terms of the Creative Commons Attribution 4.0 International License (http://creativecommons.org/licenses/by/4.0/), which permits use, sharing, adaptation, distribution and reproduction in any medium or format, as long as you give appropriate credit to the original author(s) and the source, provide a link to the Creative Commons license and indicate if changes were made.

The images or other third party material in this chapter are included in the chapter's Creative Commons license, unless indicated otherwise in a credit line to the material. If material is not included in the chapter's Creative Commons license and your intended use is not permitted by statutory regulation or exceeds the permitted use, you will need to obtain permission directly from the copyright holder.

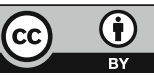

